\title{
COMPARAÇÃO ENTRE PROTOCOLOS PARA DETERMINAÇÃO DO LIMIAR ANAERÓBIO EM CANOÍSTAS
}

\section{Comparison between protocols for determination of the anaerobic threshold canoes}

\author{
Marcus Peikriszwili Tartaruga ${ }^{1}$, Fabiana Fernandes Vaz ${ }^{1}$, Marcelo Coertjens ${ }^{1}$, Caio Daniel Schmitt' \\ Carlos Leandro Tiggemann' ${ }^{1}$, Luiz Fernando Martins Kruel ${ }^{1}$
}

${ }^{1}$ Universidade Federal do Rio Grande do Sul - Escola de Educação Física - Laboratório de Pesquisa do Exercício - Grupo de Pesquisa em Atividades Aquáticas e Terrestres

\begin{abstract}
Resumo: O objetivo foi comparar e relacionar a velocidade no limiar anaeróbio (LA), determinada pelo método do lactato mínimo, com quatro velocidades críticas (VCs) determinadas nas combinações: 200/500/1000; 200/500; 500/1000 e 200/1000m. Sete canoístas de velocidade, entre 14 e 21 anos, participaram do estudo. Para comparação entre a velocidade no LA e as VCs foi realizado o teste $T$ pareado de Student e, para a verificação da relação das mesmas, o coeficiente de determinação linear $\left(r^{2}\right)$, com $p<0,05$. Não foram encontradas diferenças significativas nos valores de velocidade no LA e na VC de 200/500m, com $r^{2}=0,39$. Nas demais VCs, foram encontradas diferenças significativas: $200 / 500 / 1000 \mathrm{~m}$ e $r^{2}=0,51,500 / 1000 \mathrm{~m}$ e $r^{2}=0,46$ e 200/1000m e $r^{2}=0,52$. A VC em canoístas de velocidade, na combinação de 200/500m, pode ser utilizada na predição da velocidade no LA, podendo ser considerado um índice de avaliação e prescrição de treinamento.
\end{abstract}

Palavras-chave: lactato, limiar anaeróbio, velocidade crítica

Abstract: The objective was compare and to relate the velocity in the anaerobic threshold (LA), determined for the method of minimum lactate, with four critical velocity (VCs) determined in the combinations: 200/500/1000; 200/500; 500/1000 and 200/1000m. Seven athletes of the canoeing, between 14 and 21 years, had participated of the study. For analysis statistics the dependent test T of Student and the coefficient of linear determination $\left(r^{2}\right)$ was used, with $p<0,05$. There were no significant differences between the values of velocity in the LA and the VC of $200 / 500 \mathrm{~m}$, with $r^{2}=0,39$. In the others VCs, differences had been found significant: 200/500/1000m and $r^{2}=0,51,500 / 1000 m$ and $r^{2}=0,46$ and 200/1000m and $r^{2}=0,52$. The indirect method of determination of the VC in athletes of the canoeing, in the combination of $200 / 500 \mathrm{~m}$, can be used in the prediction of the velocity in the LA, being able to be considered an index of evaluation and prescription of the training.

Key words: lactate, anaerobic threshold, critical velocity.

Aceito em 10/08/2009 - Rev. Educ. Fís. 2009 Jun: 145: 21-27. Rio de Janeiro - RJ - Brasil

\section{INTRODUÇÃO}

Diversos procedimentos têm sido utilizados para avaliação da capacidade aeróbica de atletas de rendimento e, conseqüentemente, a elaboração de periodizações visando a melhora do condicionamento físico(1,2). A busca por um índice capaz de refletir a perfeita integração que deve existir entre os sistemas cardiovascular, respiratório e muscular para ajustar o aumento da demanda energética que existe durante o exercício tem sido o tema central de diversos estudos $^{(3,4,5)}$. Parte deste grande interesse provém das importantes implicações e aplicações que um índice desta natureza pode apresentar nas áreas da atividade física, do esporte, da clínica e da reabilitação(6).

Inicialmente, o índice que apresentou maior validade para estas aplicações e por isso foi considerado por muitos pesquisadores como padrão-ouro, foi o consumo máximo de oxigênio $\left(\mathrm{VO}_{2 \text { máx }}\right)$. Por meio de sua aferição durante um teste incremental, é possível identificar também os limiares ventilatórios, mais precisamente o segundo limiar ventilatório $\left(\mathrm{VO}_{2 \mathrm{~V} v}\right)$, 
ou ponto de compensação ventilatória, um dos mais importantes índices para a predição do desempenho aeróbico. Posteriormente, alguns estudos realizados a partir da década de 60, identificaram na resposta do lactato sangüíneo ao exercício, um outro índice de igual relevância para a avaliação aeróbica(7).

Atualmente, o limiar anaeróbio (LA) é um dos parâmetros mais usados, tanto como indicador da capacidade de desempenho físico aeróbio quanto na prescrição do treinamento ${ }^{(8)}$, existindo evidências de que o desempenho em atividades esportivas contínuas e prolongadas se correlaciona melhor com o LA do que com o $\mathrm{VO}_{2 \text { máx }}{ }^{(9,8)}$. O LA pode ser entendido como o ponto de desequilíbrio entre a produção e remoção do lactato ${ }^{(5,10)}$ no entanto, a determinação do LA através da medição do lactato sanguíneo (limiar de lactato - LL) envolve uma abordagem invasiva e com alto custo.

Visando a busca de um método da qual propicie uma abordagem não invasiva e com baixo custo na determinação do LA, surgiu o método da potência crítica (PC) como alternativa para determinação da velocidade correspondente ao $\mathrm{LA}^{(6)}$.

A base para o conceito de PC provém da existência de uma relação hiperbólica entre carga de trabalho e o tempo que cada carga é sustentada ${ }^{(11)}$. Em teoria, o cálculo da PC proporciona uma estimativa de carga que pode ser mantida por "um tempo muito longo sem o aparecimento de fadiga (12,11)" ou "teoricamente quase indefinido ${ }^{(13) "}$.

Desde o primeiro artigo elaborado por Scherrer et al. ${ }^{(14)}$ com o título "estudo do trabalho e da fadiga muscular", muitos outros têm sido dedicados à aplicação do conceito de PC a exercícios gerais ${ }^{(15)}$. Um exemplo corresponde à velocidade crítica (VC), aplicada, primeiramente, para a natação por Wakayoshi et al.(16) e, posteriormente para outras modalidades esportivas como a corrida, o remo e, até, exercícios em cadeiras de roda(15). De acordo com Wakayoshi et al. (16), a VC corresponde a mais alta intensidade de exercício que teoricamente pode ser mantida por um longo período de tempo sem exaustão, resultante da relação entre distância versus tempo. Este método apresenta muitas vantagens como a facilidade de aplicação, permite a análise de grande número de atletas, a possibilidade que as avaliações aconteçam nas sessões de treino, envolvendo um baixo custo operacional e não envolvendo variáveis bioquímicas ${ }^{(16,17)}$.

Segundo Wakayoshi et al.(16) e Kokubun(18), a VC na natação parece coincidir com o LA, definido como a velocidade de nado correspondente a uma concentração de lactato de $4 \mathrm{mM}$ no sangue em teste incremental. Em ambos os trabalhos, houve equivalência entre a VC e a velocidade correspondente ao LA, com correlações de 0,86 para Wakayoshi et al.(17) e 0,89 para Kokubun ${ }^{(18)}$. Já em exercício em cicloergômetro, estudos recentes demonstraram não existir diferenças significativas entre as estimativas de PC e a potência no $\mathrm{VO}_{2 \mathrm{lv}}{ }^{(19)}$.

Em uma revisão sobre o assunto, Gastin(20) encontrou curvas de projeção ajustada comum de contribuição aeróbica em atividades aeróbicas. As estimativas foram feitas a partir de medidas diretas de consumo de oxigênio $\left(\mathrm{VO}_{2}\right)$ e déficit de oxigênio $\left(\mathrm{DO}_{2}\right)$, mudanças nas concentrações de substratos e metabólicos musculares, e modelagens matemáticas de desempenho com base em princípios físicos (PC e VC). A canoagem, apesar de possuir características cíclicas de movimentos, é citada apenas em um estudo dentre os 116 revisados por Gastin ${ }^{(20)}$. Além disso, poucos estudos, até o momento, procuraram validar a utilização da VC na canoagem a partir da comparação com um método direto, como o limiar de lactato.

Dessa forma, o objetivo do presente estudo foi comparar e relacionar um método direto com um método indireto para determinação da velocidade no LA em canoístas de rendimento.

\section{MATERIAIS E MÉTODOS}

A amostra foi composta de 7 canoístas de velocidade, da Associação de Canoagem Guahyba, da cidade de Guaíba - RS. Todos os atletas, 
selecionados por voluntariedade, eram do sexo masculino, saudáveis e com mais de dois anos de experiência em provas de rendimento a nível regional e nacional. Os dados de caracterização da amostra encontram-se descritos na TABELA 1.

\section{TABELA 1}

MÉDIA E DESVIO-PADRÃO DAS VARIÁVEIS MASSA CORPORAL E ESTATURA DE CANOISTAS DE VELOCIDADE

\begin{tabular}{cc}
\hline VARIÁVEIS & CANOÍSTAS $(\mathbf{n}=\mathbf{7})$ \\
\hline Idade (anos) & $17,6 \pm 2,2$ \\
Massa corporal $\mathbf{( k g )}$ & $64,3 \pm 5,7$ \\
Estatura $(\mathbf{c m})$ & $170,1 \pm 3,2$ \\
\hline
\end{tabular}

A metodologia foi composta de dois testes, ambos desenvolvidos na raia da Associação de Canoagem Guahyba. O primeiro teste teve por objetivo a determinação da VC e o segundo teste, uma semana após o primeiro, a determinação da velocidade no limiar de lactato através do protocolo de lactato mínimo desenvolvido por Davis e Gass ${ }^{(21)}$. Para ambos os testes foi utilizado um caiaque K-1 de fibra de vidro, e remo de fibra de carbono.

Para a determinação da VC, foram utilizadas as distâncias de 200, 500 e 1000m, correspondentes às distâncias de treino. Os canoístas percorreram as três distâncias em máximo esforço, anotandose os respectivos tempos para a determinação das VCs nas segunintes combinações: VC1 (200-500$1000 m)$, VC2 (200-500m), VC3 (500/1000m) e VC4 (200-1000m). Foi respeitado um intervalo de 24 horas entre as distâncias percorridas. Antes de cada teste, os participantes realizavam um alongamente e um breve aquecimento. A determinação da VC foi feita através da utilização do protocolo proposto por Wakayoshi et al. ${ }^{(16)}$, adapatdo para a canoagem. A FIGURA 1 demonstra um gráfico da distância em função do tempo, desenvovido no Microsoft Excel para a determinação da VC através do coeficiente angular da reta (exemplo de determinação da VC4) para um canoísta.

\section{FIGURA 1}

GRÁFICO DA DISTÂNCIA EM FUNÇÃO DO TEMPO DE UM CANOÍSTA UTILIZADO PARA A DETERMINAÇÃO DA VC ATRAVÉS DO COEFICIENTE ANGULAR DA RETA.

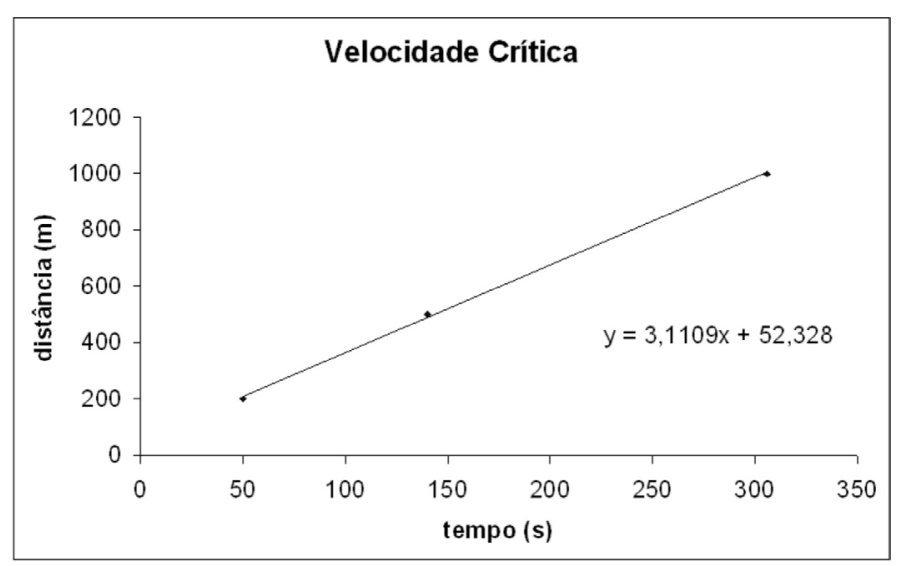

Foi utilizado o protocolo de lactato mínimo (LM) para a determinação da velocidade no LL (VLL). O mesmo consiste de um teste progressivo precedido por um exercício máximo para induzir a hiperlactacidemia. Durante os estágios iniciais deste teste progressivo, a remoção de lactato é superior à sua produção e o lactato diminui até um momento a partir do qual a produção passa a superar a remoção do mesmo, que começa a se acumular novamente(22). Assim, a carga de trabalho correspondente à menor concentração de lactato durante o teste identifica o lactato mínimo, ponto de equilíbrio entre a produção e a remoção de lactato(22), que corresponde ao limiar anaeróbio, assim como a máxima fase estável de lactato sangüíneo(23).

Para a determinação do LM, os canoístas percorreram uma distância de $200 \mathrm{~m}$ em intensidade máxima de esforço e em intensidades submáximas de esforço correspondentes a 50\%, 40\%, 30\%, 20\% e 10\% acima do tempo de performance nos $200 \mathrm{~m}$. Foram coletados $4 \mathrm{mM}$ de sangue do lóbulo da orelha direita dos atletas dez minutos antes e um minutos após o teste máximo de esforço e, no final de cada intensidade submáxima de esforço. A concentração de lactato foi analisada por um lactímetro Accutrend $®$ (Roche - Basel, Suíça).

Todos os valores de lactato sanguíneo foram plotados no Microsoft Excel para a determinação do LM e, conseqüêntemente, a velocidade no ponto de ocorrência do lactato mínimo. 
Para coletar o sangue e conseguir manter a intensidade submáxima desejada, os atletas eram acompanhados por um bote inflável com motor de $25 \mathrm{hp}$, no qual os avaliadores passavam o tempo parcial durante o percurso, informando o tempo na metade $(100 \mathrm{~m})$ e no final dos $(200 \mathrm{~m})$. Essas distâncias foram sinalizadas com boias flutuantes. Ao total foram utilizadas 3 boias (0, 100 e 200m). Antes de cada intensidade submáxima, os atletas foram informados dos tempos necessários que deveriam alcançar os 100 e $200 \mathrm{~m}$. Instantaneamente, os avaliadores analisavam se o tempo necessário nos 100 e $200 \mathrm{~m}$ era alcanado pelos atletas. Em nenhum momento houve a necessidade de se repetir alguma intensidade submáxima. Os testes foram executados entre as 8 e 12h da manhã, em água parada com vento moderado sem formação de marolas, com umidade do ar entre $60 \%$ e $80 \%$, e temperatura entre de 16 a $20^{\circ} \mathrm{C}$. Duarnte a intensidade máxima de esforço, os atletas receberam estímulos verbais para alcançarem a melhor performance nos $200 \mathrm{~m}$. Os tempos executados pelos atletas no texte máximo de esforço e as altas concentrações de lactato demonstraram que os atletas realizaram o exercício em alta intensidade.

A FIGURA 2 demonstra um gráfico da concentração de lactato em função do tempo, desenvovido para a determinação do lactato mínimo.

\section{FIGURA 2}

GRÁFICO DA CONCENTRAÇÃO DE LACTATO EM FUNÇÃO DO TEMPO DE UM CANOÍSTA UTILIZADO PARA A DETERMINAÇÃO DO LACTATO MÍNIMO.

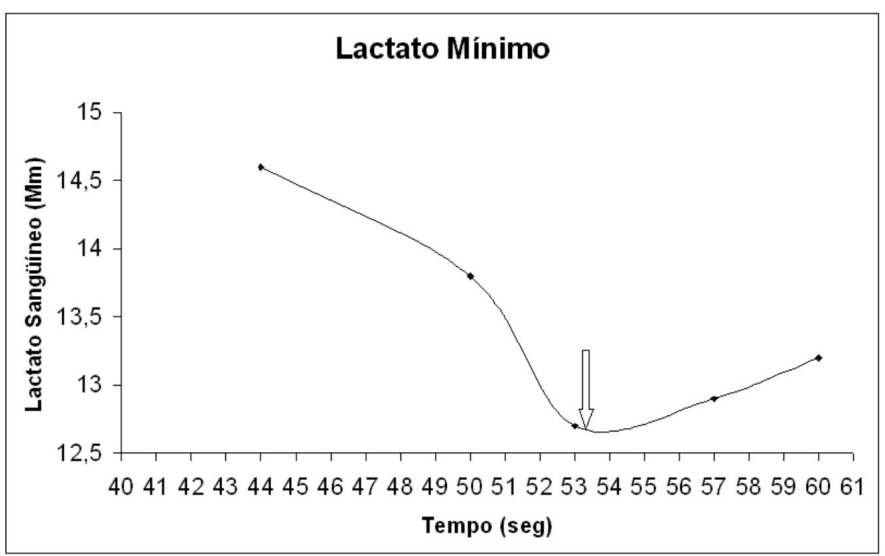

Verificou-se a normalidade dos dados referentes às velocidades analisadas (velocidade no LM e as quatro VCs) através do teste de Shapiro-Wilk. Para comparação das médias entre a velocidade no LL e as VCs foi realizado o teste " $t$ " de Student para amostras dependentes e, para a verificação da relação das mesmas, o coeficiente de determinação linear $\left(r^{2}\right)$, com $p<0,05$. Foi utilizado o pacote estatístico SPSS versão 11.0 .

\section{RESULTADOS}

A TABELA 2 apresenta os valores médios e desvio padrão das velocidades correspondentes as combinações das VCs e a velocidade correspondente ao lactato mínimo. Não foram encontradas diferenças significativas entre a VC2 e a velocidade correspondente ao lactato mínimo.

Em seguida, são demonstrados os dados dos coeficientes de determinação $\left(r^{2}\right)$ entre as VCs e a VLL (TABELA 3). Todas as relações entre VCs e VLL apresentaram comportamento direto.

\section{TABELA 2}

MÉDIA E DESVIO-PADRÃO DAS VCS DETERMINADAS A PARTIR DAS COMBINAÇÕES DAS DISTÂNCIAS DE 200, 500 E 1000M E, DA VELOCIDADE CORRESPONDENTE AO VALOR DE LACTATO MÍNIMO DE CANOÍSTAS DE VELOCIDADE

\begin{tabular}{cc}
\hline (p-valor $)$ & $\begin{array}{c}\text { CANOÍsTAS } \\
(\mathbf{n}=7)\end{array}$ \\
\hline VC1 - 200/500/1000m (0,009) & $3,33 \pm 0,33 m \cdot \mathrm{s}^{-1}$ * \\
VC2 - 200/500m (0,640) & $3,76 \pm 0,24 \mathrm{~m} \cdot \mathrm{s}^{-1}$ \\
VC3 - 500/1000m (0,003) & $3,17 \pm 0,37 \mathrm{~m} \cdot \mathrm{s}^{-1}$ * \\
VC4 - 200/1000m (0,011) & $3,36 \pm 0,32 \mathrm{~m} \cdot \mathrm{s}^{-}$* \\
VLL & $3,71 \pm 0,36 \mathrm{~m} \cdot \mathrm{s}^{-}$ \\
\hline
\end{tabular}

Velocidade crítica (VC) e Velocidade no limiar de lactato (VLL). $\left(^{*}\right)$ Diferenças significativas para $p=0,05$ entre as VCs e o LL. 


\section{TABELA 3}

COEFICIENTES DE DETERMINAÇÃO ENTRE AS VELOCIDADES CRÍTICAS E A VELOCIDADE NO LIMIAR DE LACTATO DE SETE CANOÍSTAS DE VELOCIDADE.

\begin{tabular}{cc}
\hline VARIÁVEIS & $\boldsymbol{r}^{2}$ \\
\hline VC1 (200/500/1000m) vs VLL & 0,51 \\
VC2 (200/500m) vs VLL & 0,39 \\
VC3 (500/1000m) vs VLL & 0,46 \\
VC4 (200/1000m) vs VLL & 0,52 \\
\hline
\end{tabular}

Velocidade crítica (VC), Velocidade no limiar de lactato (VLL) e Coeficiente de determinação $\left(r^{2}\right)$.

\section{DISCUSSÃO}

O objetivo deste estudo foi comparar e relacionar um método invasivo de determinação do LL, respeitando a individualidade fisiológica dos atletas, com um método não invasivo utilizando três distância fixas, principalmente porque fisiologicamente a canoagem é um esporte onde a capacidade aeróbia influencia significativamente a performance, da mesma forma como ocorre nas modalidades de meio-fundo e fundo da natação, ciclismo e corridas, da qual a VC e PC são variáveis consideradas preditoras da performance por se relacionarem com o $\operatorname{LL}^{(24,16,17)}$.

O LL, o limiar glicêmico individual, a velocidade associada ao $\mathrm{VO}_{2 \text { máx }}$ e a VC e/ou PC têm sido utilizados na avaliação aeróbia e prescrição de exercícios para atletas de natação, corrida e ciclismo ${ }^{(25,26)}$. No entanto, poucos estudos têm se utilizado da VC e da PC para determinação da capacidade anaeróbia de canoístas de rendimento( ${ }^{(6)}$.

Fontes et al.(27), verificando a aplicabilidade dos pressupostos do modelo de VC à canoagem na tentativa de estimar indiretamente a velocidade da máxima fase estável de lactato sangüíneo de oito canoístas do sexo masculino, através da realização de tiros máximos de 750, 1000 e 1500m e, da determinação da velocidade na máxima fase estável de lactato sangüíneo utilizando-se velocidades correspondentes a 98, 100 e $102 \%$, verificaram uma tendência de equilíbrio dinâmico do lactato sangüíneo quando a $98 \%$ da VC, demonstrando uma aproximação da VC com a máxima fase estável de lactato sangüíneo. No nosso estudo, não foi avaliada a máxima fase estável de lactato sanguíneo e sim, a mínima concentração de lactato sangüíneo determinada através do protocolo de lactato mínimo, onde verificamos não existir diferenças significativas na velocidade de menor concentração de lactato sangüíneo e a VC2 (200/500m), demonstrando que também a VC pode ser preditora da VLL.

De acordo com Fontes et al. ${ }^{(27)}$, quando distâncias com durações menores a 180s são utilizadas na determinação da VC na canoagem de velocidade, a mesma pode ser consideravelmente superestimada. Provavelmente, quando durações de tiros inferiores a 180s são empregadas, a inércia do sistema aeróbio em se ajustar ao seu valor de estado estável pode contribuir de forma significativa na superestimação da VC, ao causar artefato de aumento da inclinação da reta de regressão que caracteriza a relação distância-tempo ${ }^{(28)}$. No nosso estudo, as distâncias de 200 e 500 metros ocasionaram tempos menores que 180s. No entanto, justamente a VC determinada a partir das distâncias de 200 e 500m não apresentou diferença estatisticamente significativa com a VLL, Esse resultado demonstra que também a distância utilizada na determinação da máxima performance na aplicação do protocolo de lactato mínimo pode influenciar, de forma significativa, nas comparações entre a velocidade no LL e a VC. Nós utilizamos uma distância de $200 \mathrm{~m}$ para a determinação do tempo de performance. A partir do tempo de performance dos $200 \mathrm{~m}$, os atletas desenvolveram tiros submáximos correspondentes a 50,40,30, 20 e 10\% para a determinação da velocidade no ponto de inflexão da curva lactato versus tempo.

Da mesma, Toubekis et al. ${ }^{(29)}$ destacam que a escolha nas distâncias para a determinação da VC de jovens nadadores podem influenciar na 
relação das VCs com a VLL. Os autores destacam que grandes distâncias, a partir de $1000 \mathrm{~m}$, podem subestimar a VC, devido a fatores relacionados a desidratação, depleção de glicogênio, fadiga e queda motivacional.

Sendo assim, por convenção, a maioria dos pesquisadores recomenda cargas preditivas variando entre 2 e 10 minutos como sendo ótimas, visando assim minimizar o erro associado às estimativas. Portanto, para se conseguir resultados adequados e com alto grau de precisão, a escolha cuidadosa das cargas preditivas parece ser fundamental, independente da modalidade esportiva(11). No nosso estudo, foram escolhidas as distâncias de 200, 500 e $1000 \mathrm{~m}$ devido a utilização destas no treinamento desportivo da nossa amostra.

\section{CONCLUSÃO}

Podemos concluir que o método indireto de determinação da VC em canoístas de velocidade, na combinação de 200/500m, pode ser utilizado na predição da velocidade no LA, podendo ser considerado um índice de avaliação e prescrição de treinamento.

Como aplicação prática, recomendamos a utilização da VC na combinação de 200/500m para predizer a velocidade no LA e, conseqüentemente, auxiliar na elaboração da periodização do treinamento em canonistas de velocidade, que possuam semelhantes características fisiológicas (idade, massa e estatura).

Como limitação deste estudo, gostaríamos de enfatizar a falta de um número maior de participantes. Sugere-se, também, para futuros estudos, a utilização, se possível, de um ergoespirômetro portátil com a finalidade de investigar os comportamentos dos parâmetros ventilatórios também.

\section{REFERÊNCIAS BIBLIOGRÁFICAS}

1. Bosquet $L$, Leger $L$, Legros $P$. Methods to determine aerobic endurance. Sports Med 2002;32(11):675-700.
2. Smith DJ, Norris SR Hogg JM. Performance evaluation of swimmers: scientific tools. Sports Med 2002;32(9):539-54.

3. Astrand PO. Human physical fitness with special reference to sex and age. Physiol Rev 1956;36(3):307-35.

4. Noakes TD. Implications of exercise testing for prediction of athletic performance: a contemporary perspective. Med Sci Sports Exerc 1988;20(4):319-30.

5. Coyle EF. Integration of the physiological factors determining endurance performance ability. Exerc Sport Sci Ver 1995:23:25-63.

6. Bunioto SH. Potência crítica: uma revisão de um conceito. Centro de Estudos de Fisiologia do Exercício 2005:1-8.

7. Denadai BS. Avaliação aeróbia: determinação indireta da resposta do lactato sanguíneo. Motrix 2000:1-24.

8. Svedahl K, Macintosh BR. Anaerobic threshold: the concept and methods of measurement. Can J Appl Physiol 2003;28(2):299-323.

9. Ribeiro JP. Limiares metabólicos e ventilatórios durante o exercício. Aspectos fisiológicos e metodológicos. Arq Bras Cardiol 1995;64:171-81.

10. Hollmann W. 42 years ago-development of the concepts of ventilatory and lactate threshold. Sports Med 2001;31(5):315-20.

11. Hill DW. The critical power concept. A review. Sports Med 1993;16(4):237-54.

12. Monod $\mathrm{H}$, Scherrer J. The work capacity of synergic muscle group. Ergonomics 1965;8:329-38.

13. Moritani T, Nagata A, Devries HA, Muro M. Critical power as a measure of physical work capacity and anaerobic threshold. Ergonomics 1981;24(5):339-50.

14. Scherrer J, Samson M, Paleologue A. Étude du travail musculaire et de la fatigue. J Physiol 1954;46:887-916.

15. Vandewalle $H$, Vautier JF, Kachouri $M$, Lechevalier $\mathrm{JM}$, Monod $\mathrm{H}$. Work-exhaustion time relationships and the critical power concept. A critical review. J Sports Med Physl Fitness 1997;37(2):89-102.

16. Wakayoshi K, Ikuta K, Yoshida T, Udo M, Moritani T, Mutoh Y, Miyashita M. Determination and validity of critical velocity as an index of swimming performance in the competitive swimmer. Eur J Appl Physiol Occup Physiol 1992a;64(2):153-7. 
17. Wakayoshi K, Yoshida T, Udo M, Kasai T, Moritani T, Mutoh Y, Miyashita M. A simple method for determining critical speed as swimming fatigue threshold in competitive swimming. Int J Sports Med 1992b;13(5):367-71.

18. Kokubun E. Velocidade crítica com estimador do limiar anaeróbio na natação. Revista Paulista de Educação Física 1996;10:5-20.

19. Dekerle J, Baron B, Dupont L, Vanvelcenaher J, Pelayo P. Maximal lactate steady state, respiratory compensation threshold and critical power. Eur J Appl Physiol 2003;89(34):281-8.

20. Gastin PB. Energy system interaction and relative contribution during maximal exercise. Sports Med 2001;31(10):725-41.

21. Davis HA, Gass GC. Blood lactate concentrations during incremental work before and after maximum exercise. $\mathrm{Br} \mathrm{J}$ Sports Med 1979;13(4):165-9.

22. Tegtbur U, Busse MW, Braumann KM. Estimation of an individual equilibrium between lactate production and catabolism during exercise. Med Sci Sports Exerc 1993;25(5):620-7.

23. Tegtbur U, Machold H, Meyer H, Storp D, Busse MW. Determining the extent of intensive physical performance in patients with coronary heart disease. Z Kardiol 2001;90(9):637-45.

24. Housh TJ, Devries HA, Housh DJ, Tichy MW, Smyth $K D$, Tichy AM. The relationship between critical power and the onset of blood lactate accumulation. J Sports Med Phys Fitness 1991;31(1):31-6.
25. Stegmann H, Kindermann W, Schnabel A. Lactate kinetics and individual anaerobic threshold. Int J Sports Med 1981;2(3):160-5.

26. Silva LGDM, Pacheco ME, CampbelL CSG, Baldissera V, Simões HG. Comparação entre protocolos diretos e indiretos de avaliação da aptidão aeróbia em indivíduos fisicamente ativos. Rev Bras Med Esp 2005;11(4):210-23.

27. Fontes EB, Nakamura FY, Gobbo LA, Altimari LR, Mello JCD, Carvalho FO, Okano AH, Borges TO, Silva SGD, Cyrino ES. A velocidade crítica representa a máxima fase estável de lactato sangüíneo na canoagem? Departamento de Educação Física, Universidade Estadual de Londrina, Londrina - PR, 2002

28. Vandewalle H, Kapitaniak B, Grun S, Raveneau S, Monod H. Comparison between a 30-s all-out test and a time-work test on a cycle ergometer. Eur J Appl Physiol Occup Physiol 1989;58(4):375-81.

29. Toubekis AG, Tsami AP, Tokmakidis SP. Critical velocity and lactate threshold in young swimmers. Int J Sports Med 2006;27(2):117-23.

\section{Endereço para correspondência e pedidos de reprint:}

Para: Marcus Peikriszwili Tartaruga Laboratório de Pesquisa do Exercício - EsEF / UFRGS Rua Felizardo, 750, Jardim Botânico, Porto Alegre / RS CEP: $90690 / 200$

Tel / Fax: (51) 33085820 / (51) 33085842

E-mail: mtartaruga@bol.com.br 